\title{
Testing Results of Plasma Spraying Ceramics Coatings by Pulse Plasma Modulation Technology
}

\author{
Baruch Boris Gutman \\ Plasma Project, USSR-Israel-SUNY, Nassau Community College, NY, USA
}

\section{Email address:}

bg30002001@yahoo.com

\section{To cite this article:}

Baruch Boris Gutman. Testing Results of Plasma Spraying Ceramics Coatings by Pulse Plasma Modulation Technology. American Journal of Nano Research and Applications. Vol. 5, No. 4, 2017, pp. 49-60. doi: 10.11648/j.nano.20170504.12

Received: July 31, 2017; Accepted: August 11, 2017; Published: September 13, 2017

\begin{abstract}
The work below was done mainly in the USSR (Tajikistan) and partly continued in Israel. This technology provides substantially better coating performance compared with the respective individual processes (plasma, Cold Spraying, arc or oxy-fuel, etc.). The coatings of large areas can be accomplished at much higher quality and deposition rates than conventional plasma, arc or flame spray techniques and at a far lower cost. These benefits have been accomplished through a specially designed torch head and power supply modulation unit. The system does not require expensive argon-helium based plasma of hydrogen, nitrogen or helium mixtures but rather uses air plasma alone or in combination with hydrocarbon fuel. The plasma chemical technology overcomes a previously fundamental limitation of air plasma systems - oxidation of the coating. In addition, the proprietary design of the torch head and supply modulation unit produces both superior coating and substantially reduces the erosion rate of expensive torch electrodes thereby significantly increasing the resource of operating time of a plasma generator. Detailed design specifications and process validation experiments have been of successfully completed, and the design, technology and market of a pre-production prototype has passed through an evaluation by potential strategic partners with the impressive estimation.
\end{abstract}

Keywords: Pulse Plasma Spraying, DC Torch Shock Wave Spraying Technology, DC Arc Pulse Modulation, Pulse Modulation

\section{Introduction}

Testing of pulse plasma spraying technology [1-13] ("subtractive", + "addition" pulse modulation) by SULZER METCO AG Company/Praxair were shown in the report below and was "strictly confidential" for a long time.

The results of the first phase of collaboration between Plasma Model Ltd (Dr. B. Gutman project) and SULZER METCO were presented in the report 97PE149A composed by Dr. G. Barbezat.

During the meeting, the execution of a second series of test was decided and samples were delivered to Sulzer's Metco laboratory in city Wohlen of Switzerland.

THE PRESENT REPORT SHOW THE IMPRESSIVE POTENTIAL OF THE TECHNOLOGY OF SDr. B. GUTMAN.

\section{Material Used}

All ceramics were sprayed using bond coat M450 NS (NiA15). The following powder materials were used:

AMDRY $6060 \mathrm{Al}_{2} \mathrm{O}_{3}$ AMDRY $6420 \mathrm{Cr}_{2} \mathrm{O}_{3}$ AMDRY $6254 \mathrm{Al}_{2} \mathrm{O}_{3} 40 \% \mathrm{TiO}_{2}$

\section{Results of the Investigations}

The results of the investigations are summarized in the figure 1. All the investigated samples shows a good quality of coating.

Some samples of $\mathrm{Al}_{2} \mathrm{O}_{3}$ and $\mathrm{Cr}_{2} \mathrm{O}_{3}$ can be considered as excellent.

All samples are characterized by low porosity level, relative high hardness and reasonable bond strength, especially the samples PM41 $\left(\mathrm{Cr}_{2} \mathrm{O}_{3}\right)$, PM46 $\left(\mathrm{Al}_{2} \mathrm{O}_{3}\right)$ and PM42 $\left(\mathrm{Al}_{2} \mathrm{O}_{3} 40 \% \mathrm{TiO}_{2}\right)$. 
The influence of the spray distance on the micro hardness and the bond strength is shown in the figure 2 .

The process show only a low sensitivity to the spray distance regarding the coating properties. However the deposition efficiency is significantly affected by the spray distance.

\begin{tabular}{|c|c|c|}
\hline \multicolumn{3}{|c|}{ DE versus spray distance for A6060 } \\
\hline Spray distance [mm] & DE [\%] & Appreciation \\
\hline 200 & 79 & Excellent \\
250 & 62 & Medium \\
300 & 34 & Low \\
\hline
\end{tabular}

\begin{tabular}{|c|c|c|c|c|c|}
\hline Tear ko & Wheria & $\begin{array}{c}\text { Porpility } \\
\text { pl] }\end{array}$ & Gualy & $\begin{array}{c}\text { Ecrohardnesi } \\
\text { Hyh }\end{array}$ & $\begin{array}{l}\text { Bend thength } \\
\text { MPa! }\end{array}$ \\
\hline BA-5013/PRWD & 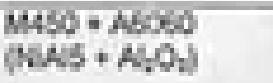 & 2 & $\mathrm{H}$ & $10 \%$ & HO \\
\hline 60-गЫ4 / PMA & $\begin{array}{l}\text { MHS + ABND } \\
\text { WhS + CrOH }\end{array}$ & 2 & Hph & 154 & 40 \\
\hline A-Fots/PM2 & 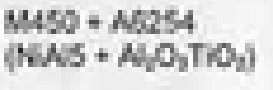 & 1 & Hgh & 1045 & 57 \\
\hline 909016/PM43 & M4S + MNOS & 2 & Cood & 918 & HS \\
\hline 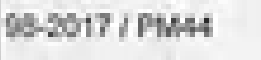 & U4S + ANos & 2 & Hp & 1ton & $+\infty$ \\
\hline 45014JPMS & 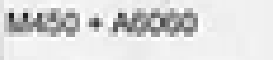 & 7 & utum & 925 & sS \\
\hline 94SOITIPM⿻ & $145+\operatorname{Mot}$ & 25 & $\mathrm{H} F$ & 1131 & 75 \\
\hline 0.200د IFAT & H45 + MODO & 2 & $\mathrm{Hy}$ & $112 t$ & $\rightarrow S$ \\
\hline 902004 JFW46 & $4450+10050$ & 25 & Modum & 14t & 45 \\
\hline 9040mirmis & M450 + M440 & 25 & Hyh & 140 & 49 \\
\hline POALG I IMLO & MAD + FLAS & 4 & ilth & 4t6ㅂ & 44 \\
\hline 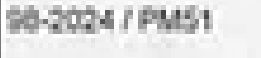 & MOD + MONO & 25 & Dond & 1004 & 34 \\
\hline 74.702s ipusa & MHO + MALO & 3 & Muthr & 1257 & Let \\
\hline 40-2020 rpust & MHS - AMSA & 1 & Hog & $m$ & $y$ \\
\hline
\end{tabular}

Figure 1. Summary of the test results of ceramics layers. 
SULZER METCO

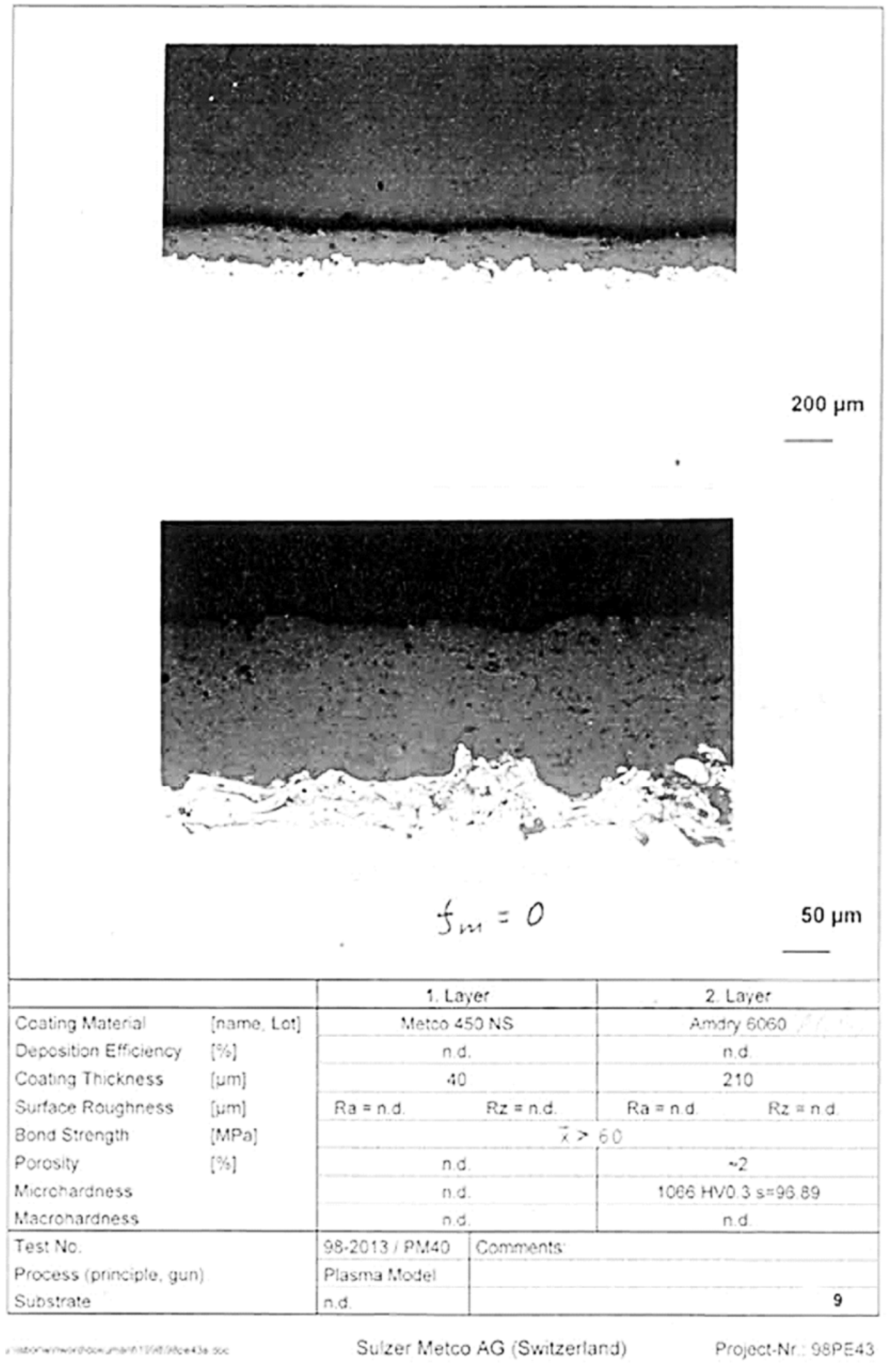

Spraying without DC arc modulation 


\begin{tabular}{|c|c|c|c|c|c|c|}
\hline \multirow[t]{2}{*}{$\begin{array}{l}\text { Spray Distanco } \\
{[\mathrm{mm}]}\end{array}$} & \multicolumn{2}{|c|}{$\begin{array}{c}\text { AMDRY } 6060 \\
\mathrm{Al}_{2} \mathrm{O}_{2}\end{array}$} & \multicolumn{2}{|c|}{$\begin{array}{c}\text { AMDRY } 6420 \\
\mathrm{Cr}_{2} \mathrm{O} \text {, }\end{array}$} & \multicolumn{2}{|c|}{$\begin{array}{l}\text { AMDRY } 6254 \\
\mathrm{AN}_{3} \mathrm{O}_{2} 40 \mathrm{TiO}_{2}\end{array}$} \\
\hline & $\mathrm{HV}_{0, \mathrm{~s}}$ & $\begin{array}{l}\text { Bond strongth } \\
\text { [MPa] }\end{array}$ & $\mathrm{HV}_{0 \mathrm{~S}}$ & $\begin{array}{l}\text { Bond strength } \\
\text { [MPa] }\end{array}$ & $\mathrm{HV}_{03}$ & $\begin{array}{l}\text { Bond strongth } \\
{[\mathrm{MPa}]}\end{array}$ \\
\hline Short 200 & 1066 & $>60$ & 1547 & 40 & 1046 & 57 \\
\hline Medium 250 & 941 & 45 & 1420 & 43 & 1001 & 48 \\
\hline Long 300 & 1004 & 33 & 1357 & 60 & 999 & 34 \\
\hline
\end{tabular}

Figure 2. Influence of the spray distance on the micro hardness and the bond strength.

\section{SULZER METCO}

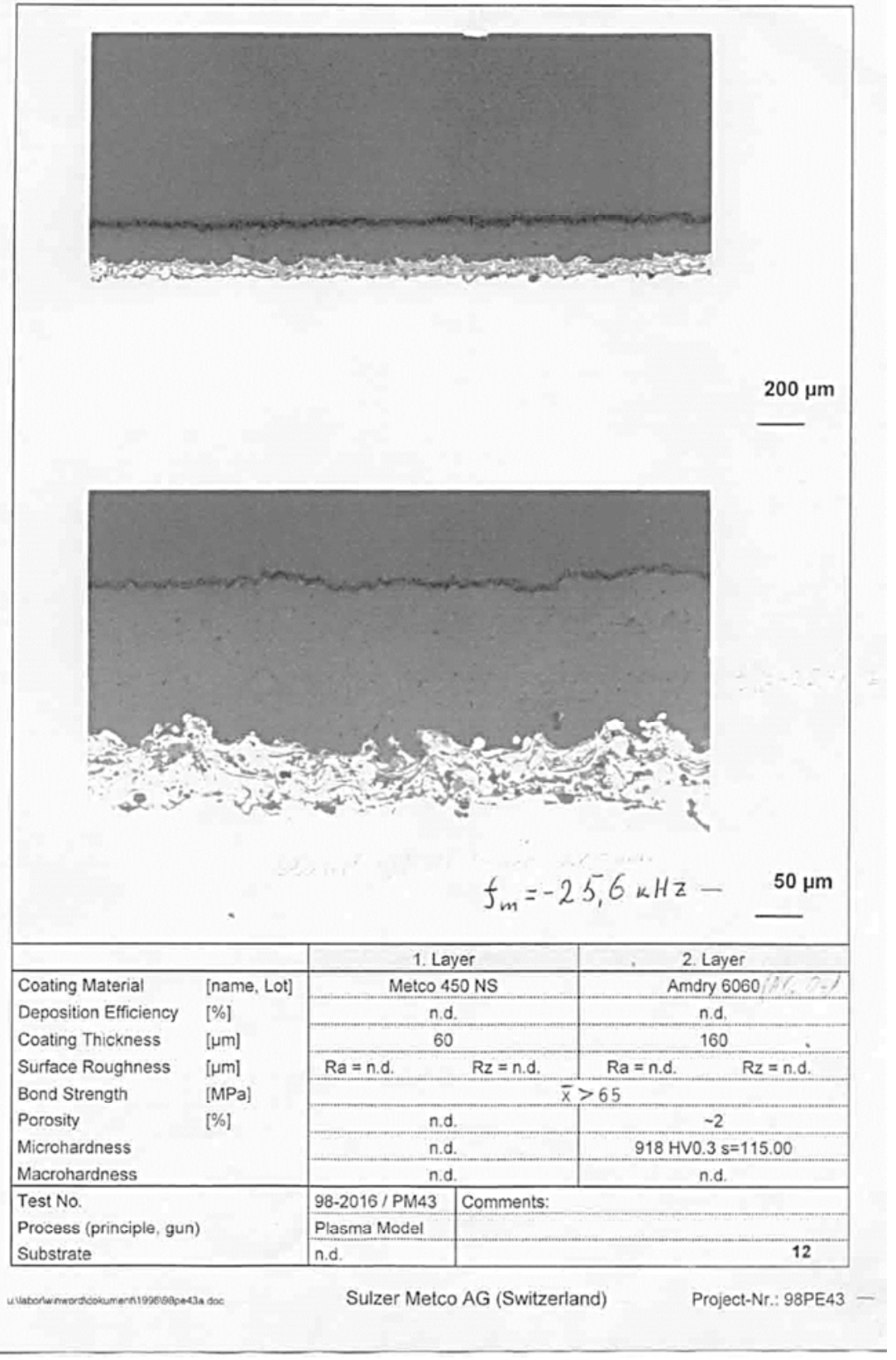

Sprayed with modulation frequency $\sim-25.6 \mathrm{kHz}$ 


\section{SULZER METCO}

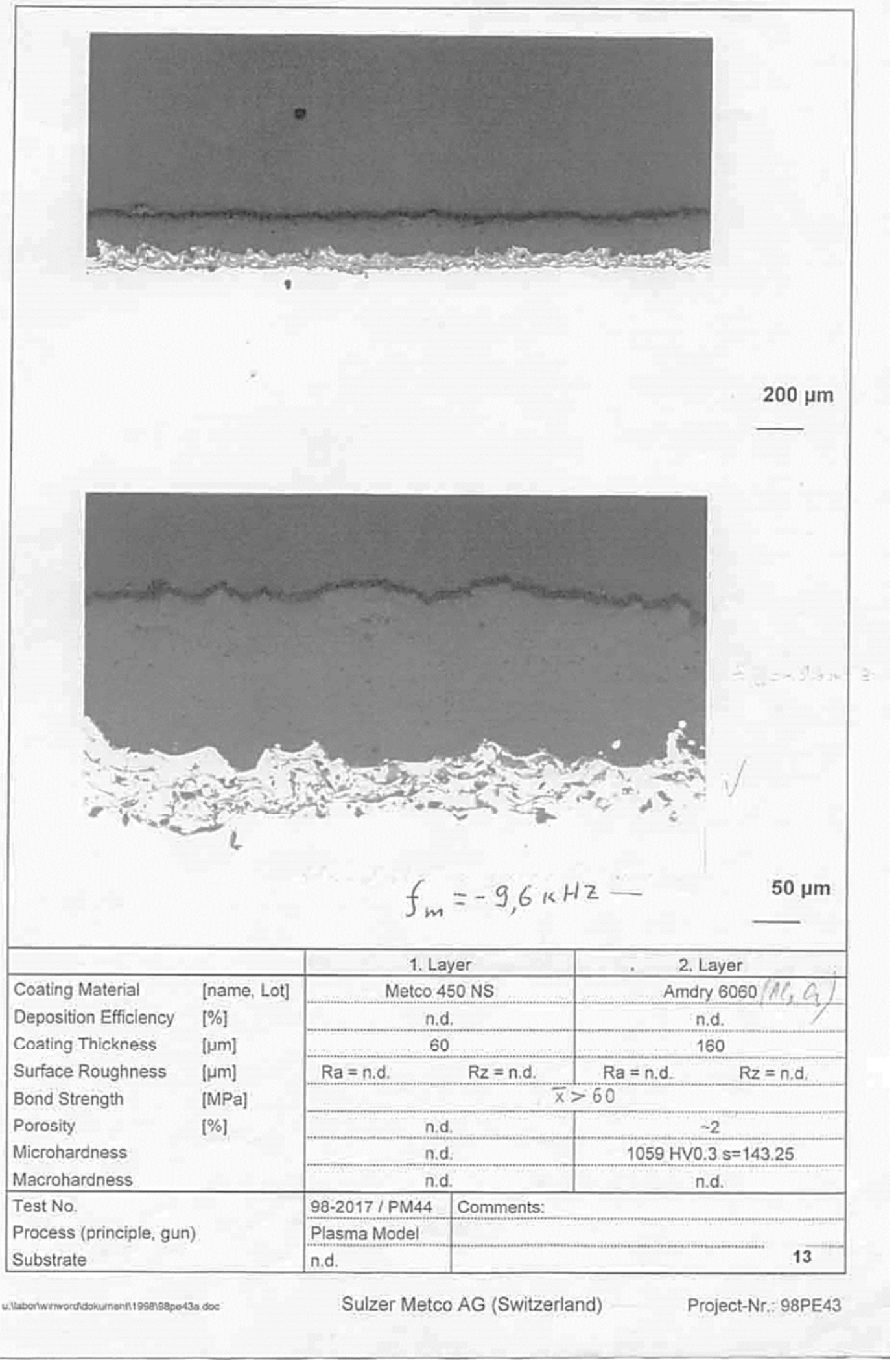

Sprayed with modulation frequency $\sim 9.6 \mathrm{kHz}$. 
SULZER METCO

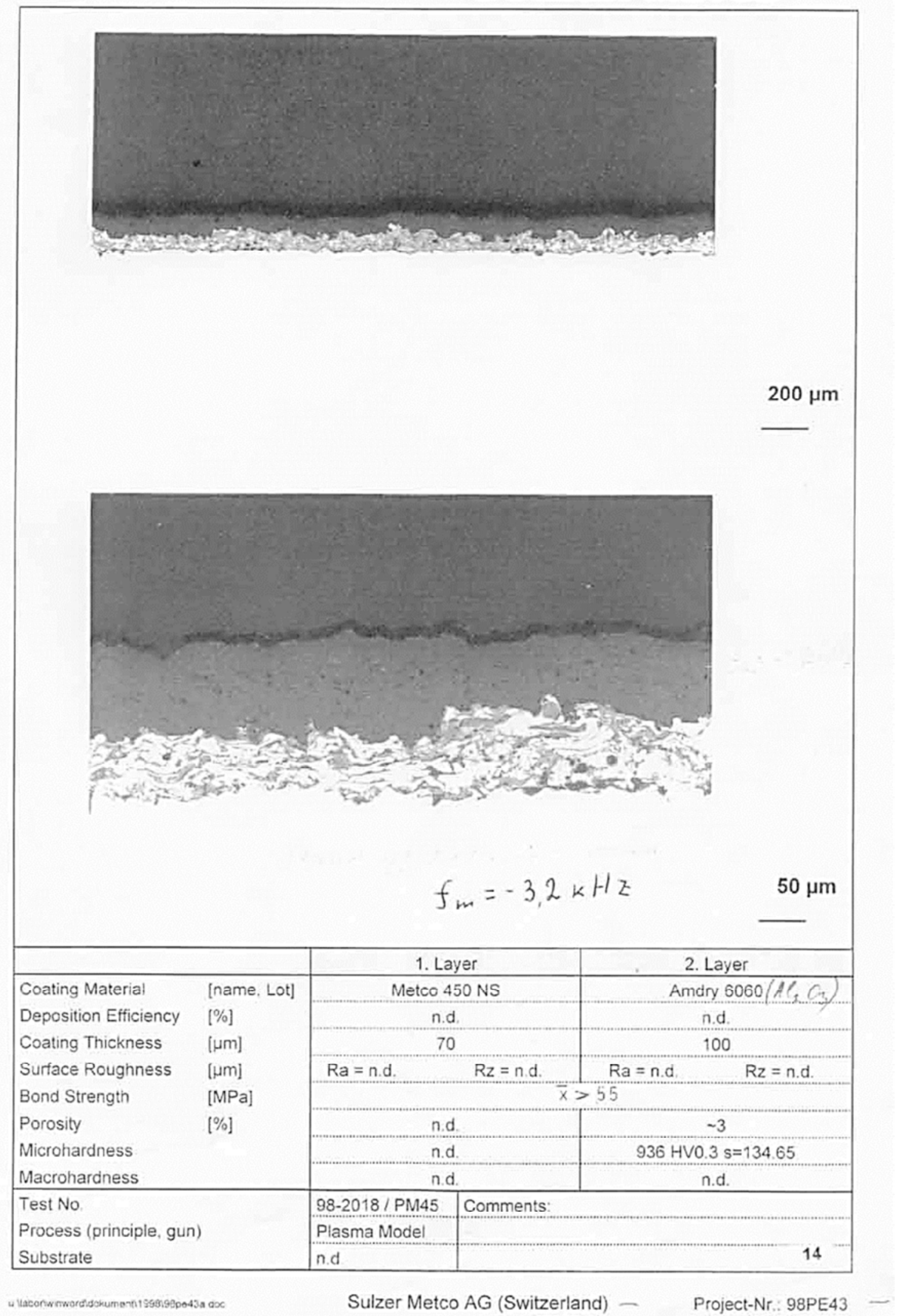

Sprayed with modulation frequency $\sim-3.2 \mathrm{kHz}$. 


\section{SULZER METCO}

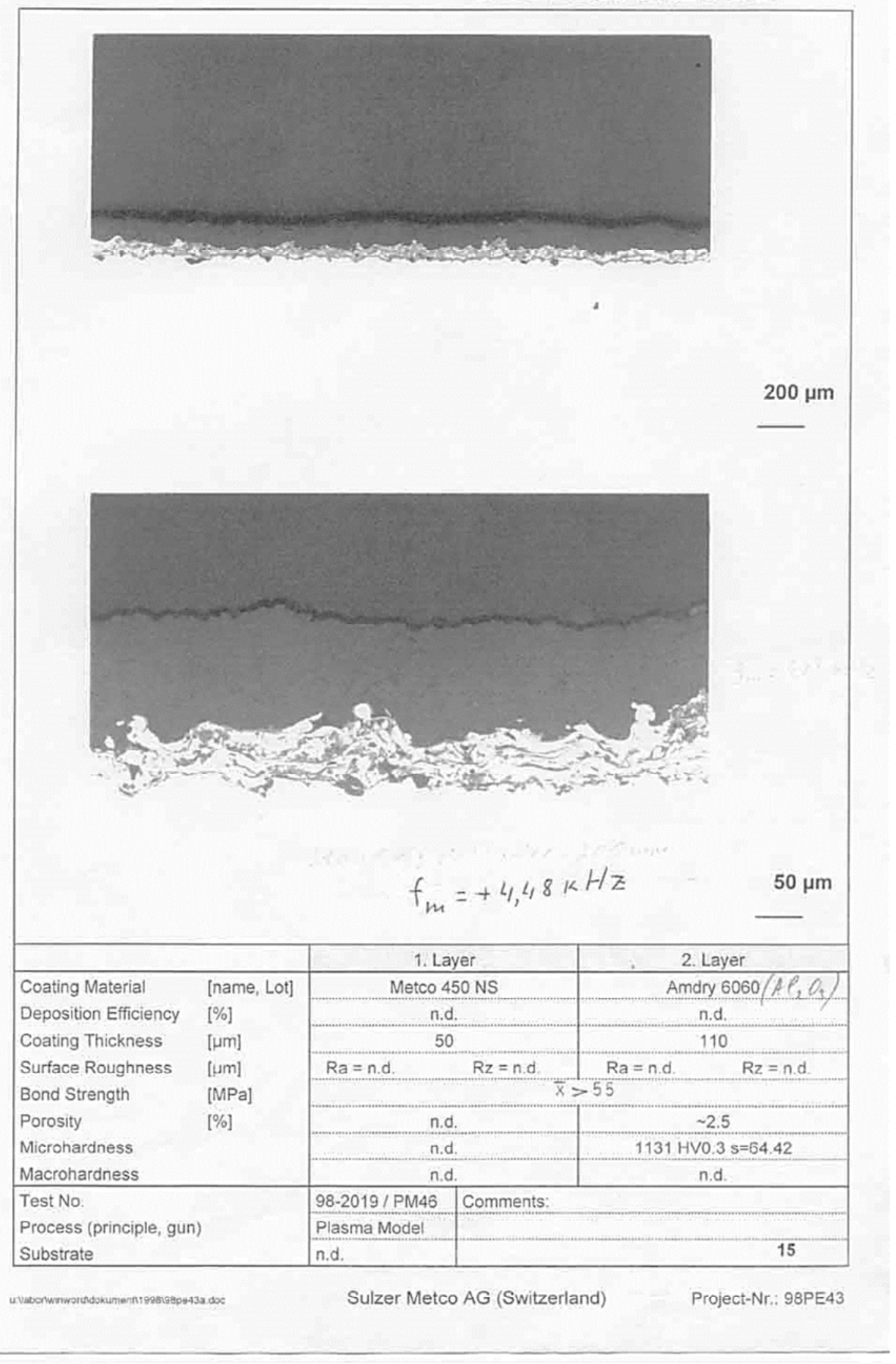

Sprayed with modulation frequency $\sim+4.4 \mathrm{kHz}$. 
SULZER METCO

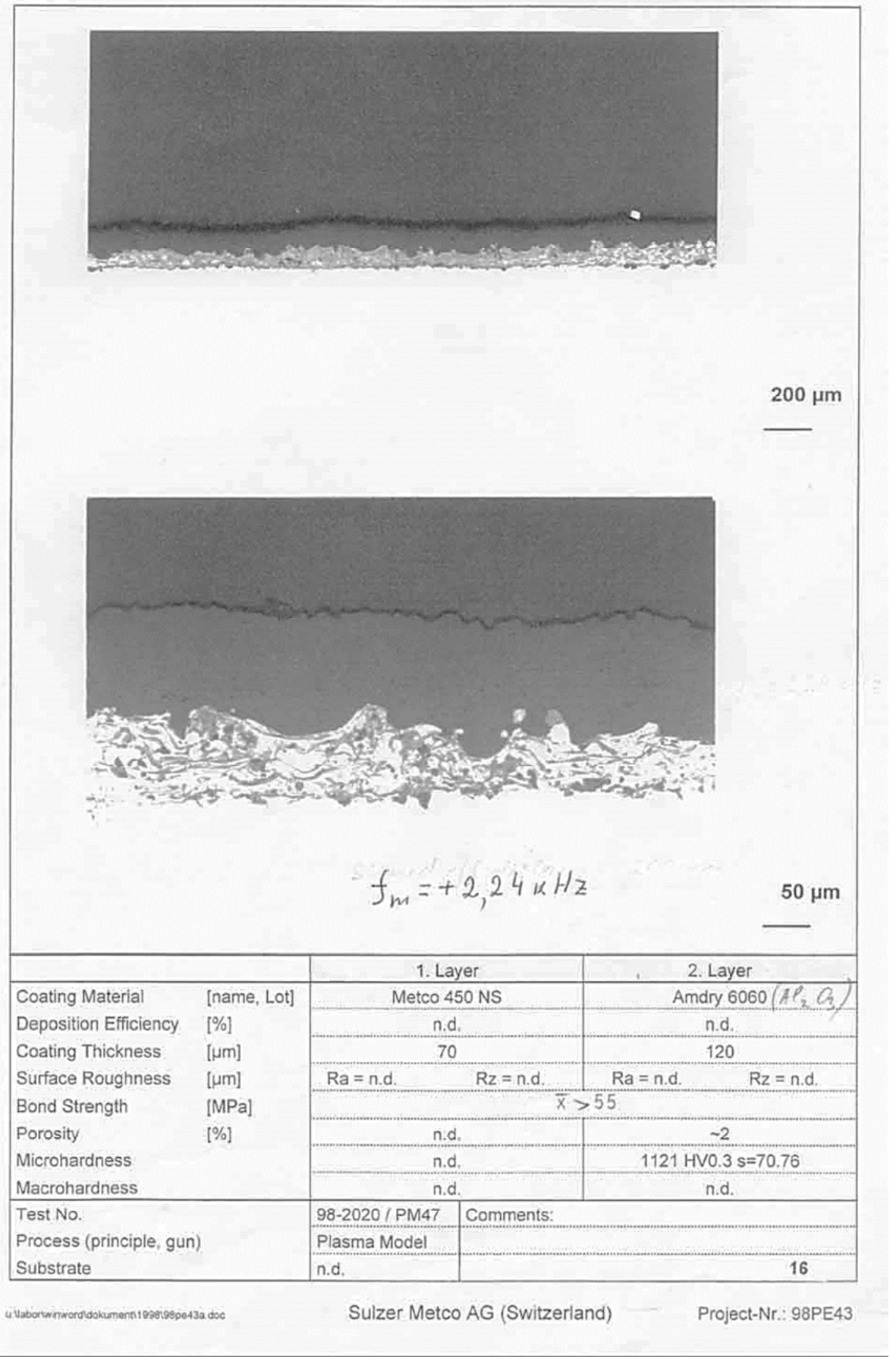

Sprayed with modulation frequency $\sim+2.24 \mathrm{kHz}$. 


\section{SULZER METCO}

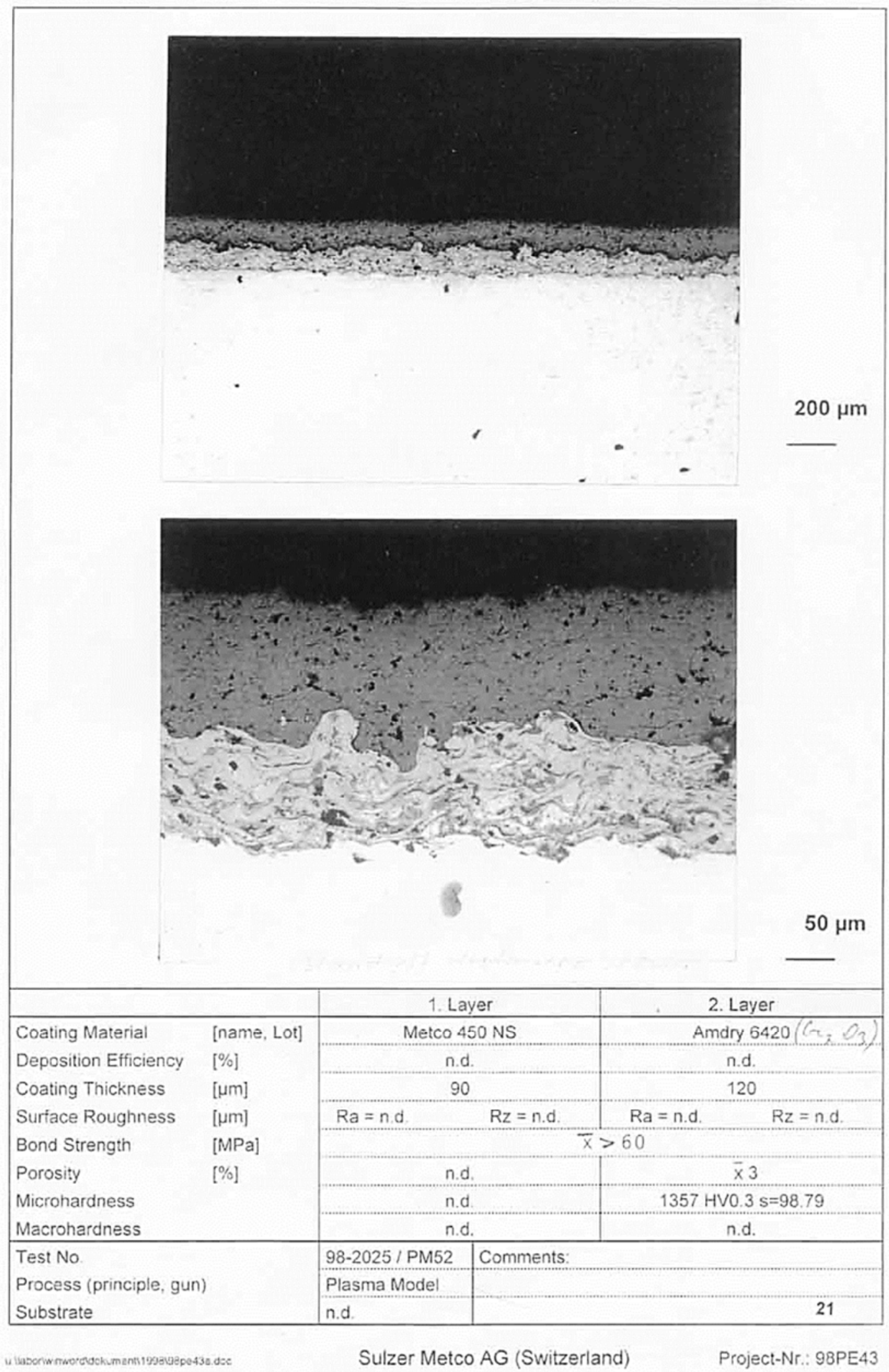

The sample having received without DC Arc modulation 


\section{SULZER METCO}

\section{Bond Strength Test Characterisation}

(DIN EN 582 "Ermittlung der Haftzugfestigkeit")

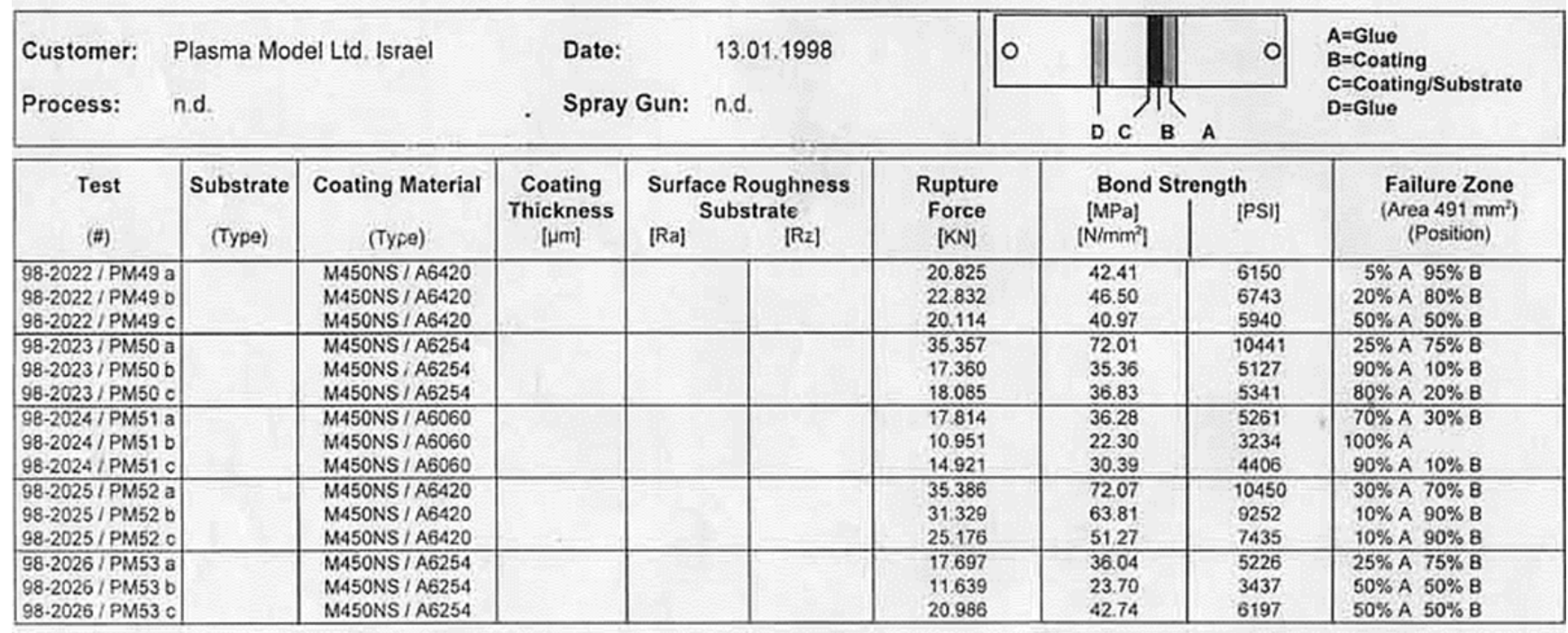

\section{SULŻER METCO}

Bond Strength Test Characterisation

(DIN EN 582 'Ermattiung der Haftzugfestigkeir')

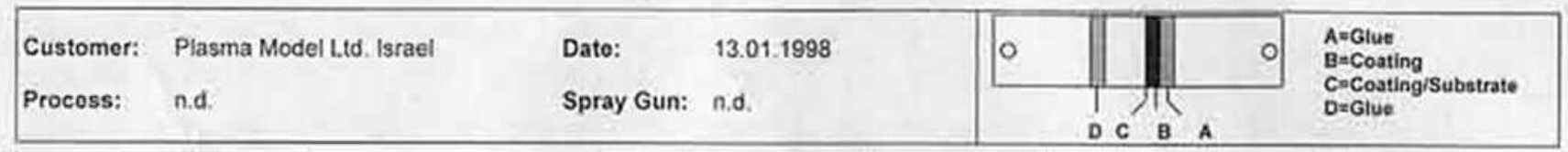

\begin{tabular}{|c|c|c|c|c|c|c|c|c|}
\hline Test & $\begin{array}{c}\text { Substrate } \\
\text { (Type) }\end{array}$ & $\begin{array}{c}\text { Coating Material } \\
\text { (Typo) }\end{array}$ & $\begin{array}{l}\text { Coating } \\
\text { Thickness } \\
\mid(\mathrm{nm} \mid\end{array}$ & $\begin{array}{l}\text { Surface Roughness } \\
\text { Substrato } \\
\text { [Ra) [Rz] }\end{array}$ & $\begin{array}{l}\text { Rupture } \\
\text { Forco } \\
\text { [iou] }\end{array}$ & $\begin{array}{l}\text { Bond } \\
{[\mathrm{MPa}]} \\
{[\mathrm{N} / \mathrm{mm}]}\end{array}$ & [PSI] & $\begin{array}{c}\text { Fallure Zono } \\
\text { (Area } 491 \mathrm{~mm}^{2} \text { ) } \\
\text { (Position) }\end{array}$ \\
\hline $\begin{array}{l}98-2013 / P M 40 \text { a } \\
98-2013 / P M 40 \text { b } \\
98-2013 / P M 40 \text { c }\end{array}$ & & $\begin{array}{l}\text { M450NS / ASO60 } \\
\text { M450NS I AGC60 } \\
\text { M4SONS I AGS660 }\end{array}$ & & & $\begin{array}{l}30088 \\
38288 \\
27307\end{array}$ & $\begin{array}{l}61.30 \\
71.98 \\
56.62\end{array}$ & $\begin{array}{c}6888 \\
11307 \\
8064\end{array}$ & $\begin{array}{l}80 \% \mathrm{~A} 20 \% \mathrm{~B} \\
100 \% \mathrm{~A} \\
100 \% \mathrm{~A}\end{array}$ \\
\hline $\begin{array}{l}96-2014 / P M 41 \text { a } \\
98-2014 / P M / 18 \\
08-2014 / P M 4 / C\end{array}$ & & 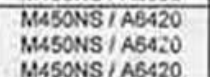 & & & $\begin{array}{l}24333 \\
15.543 \\
16400\end{array}$ & $\begin{array}{l}49.66 \\
31.66 \\
33.49\end{array}$ & $\begin{array}{l}7186 \\
4590\end{array}$ & $\begin{array}{l}10 \% \text { A } 90 \% \text { B } \\
95 \% \text { A } 5 \% \mathrm{~B} \\
25 \% \text {. }\end{array}$ \\
\hline $\begin{array}{l}68-2015 / P M 42 \text { a } \\
98-2015 / P M 42 \mathrm{~b} \\
58-2015 / P M 42 \mathrm{c}\end{array}$ & & $\begin{array}{l}\text { M45ONS I AS254 } \\
\text { M45ONS IA6254 } \\
\text { MA45ONS I AE2554 }\end{array}$ & & & $\begin{array}{l}33.058 \\
22.033 \\
13.976\end{array}$ & $\begin{array}{l}69.10 \\
44.87 \\
28.46\end{array}$ & $\begin{array}{l}10028 \\
6507 \\
4127\end{array}$ & $\begin{array}{l}80 \% \text { A } 20 \% 8 \\
90 \% \text { A } 10 \% 8 \\
100 \% \text { A }\end{array}$ \\
\hline $\begin{array}{l}98-2016 / P M 43 \text { a } \\
98-2016 / P M 43 \text { b } \\
96-2016 / P M 43 \mathrm{C}\end{array}$ & & 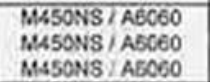 & & & $\begin{array}{l}32.112 \\
18.239 \\
32.353\end{array}$ & $\begin{array}{l}65.40 \\
33.07 \\
65.89\end{array}$ & $\begin{array}{l}0483 \\
4796 \\
0554\end{array}$ & $\begin{array}{l}100 \% A \\
100 \% \mathrm{~A} \\
100 \% \mathrm{~A}\end{array}$ \\
\hline $\begin{array}{l}98-2017 / \text { PM44 a } \\
98-2017 / \text { PM44 b } \\
05-2017 / \text { PM } 44 \text { c }\end{array}$ & & $\begin{array}{l}\text { MA5ONS/A6060 } \\
\text { MUSONS / A6060 } \\
\text { MA5ONS/AGC60 }\end{array}$ & & & $\begin{array}{l}32.698 \\
10.598 \\
10.980\end{array}$ & $\begin{array}{l}66.59 \\
33.81 \\
22.36\end{array}$ & $\begin{array}{l}0656 \\
4902 \\
3243\end{array}$ & $\begin{array}{l}70 \% \hat{A} 30 \% B \\
100 \% \hat{A} \\
90 \% A \text {. } 10 \% B \text { B }\end{array}$ \\
\hline $\begin{array}{l}98-2018 \text { / FM45 a } \\
08-2018 \text { / FM45 b } \\
95-2018 \text { / PM45 C }\end{array}$ & & $\begin{array}{l}\text { MA5ONS I ACO60 } \\
\text { MU5ONS / A6000 } \\
\text { MASONS T AEOCO }\end{array}$ & & & $\begin{array}{l}15.858 \\
29.021 \\
27.080\end{array}$ & $\begin{array}{l}32.30 \\
59.11 \\
56.15\end{array}$ & $\begin{array}{l}4683 \\
8570 \\
7997\end{array}$ & $\begin{array}{l}95 \% \text { A } 5 \% \text { B } \\
90 \% \text { A } 10 \% \text { B } \\
00 \% \text { A } 10 \% \text { B }\end{array}$ \\
\hline $\begin{array}{l}98-2019 / \text { PM46 a } \\
98-2019 / \text { PM46 b } \\
98-2019 \text { / PM46 c }\end{array}$ & & $\begin{array}{l}\text { MASONS I ABO60 } \\
\text { MASONS I AGO60 } \\
\text { MASONS I ABOED }\end{array}$ & & & $\begin{array}{l}28.442 \\
13.469 \\
22.121 \\
\end{array}$ & $\begin{array}{l}37.93 \\
39.65 \\
45.05\end{array}$ & $\begin{array}{l}8399 \\
5750 \\
6533\end{array}$ & $\begin{array}{l}60 \% \mathrm{~A} 40 \% \mathrm{~B} \\
100 \% \mathrm{~A} \\
100 \% \mathrm{~A}\end{array}$ \\
\hline $\begin{array}{l}08-2020 / \text { PMA } 7 \text { a } \\
98-2020 / \text { PNA7 b } \\
98-2020 / \text { PM47 c }\end{array}$ & & $\begin{array}{l}\text { MASONS / A6060 } \\
\text { MA5ONS / AGO60 } \\
\text { MASONS / AGOED }\end{array}$ & & & $\begin{array}{l}22.744 \\
29.263 \\
18.026\end{array}$ & $\begin{array}{l}46.32 \\
59.60 \\
36.71\end{array}$ & $\begin{array}{l}6717 \\
6042 \\
5323\end{array}$ & $\begin{array}{l}100 \% \text { A } \\
00 \% \text { A } 10 \% \mathrm{~B}\end{array}$ \\
\hline 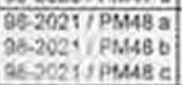 & & $\begin{array}{l}\text { MS5ONS / AG060 } \\
\text { MUSONS / A } 5060 \\
\text { M45ONS I A6060 }\end{array}$ & & & $\begin{array}{l}28.303 \\
17.8 \% 4 \\
12.533\end{array}$ & $\begin{array}{l}57.64 \\
36.28 \\
25.53\end{array}$ & $\begin{array}{l}8358 \\
5261 \\
3701\end{array}$ & $\begin{array}{r}00 \% \mathrm{~A} \quad 10 \% \mathrm{~B} \\
90 \% \mathrm{~A} 10 \% \mathrm{~B} \\
100 \% \mathrm{~A}\end{array}$ \\
\hline
\end{tabular}

\% SULZER METCO AG (Switzerland)

Ras foos-032 J Revinar o

Figure 3. Bond strenth test characterization

During my communications with the leadership of a plasma spraying company, it was revealed that their experiments with pulse Arc spraying did not provide them their wishful results, because their experiments have been being done not correctly.

Our experiments carried out by plasma forming gas Air + 
hydrocarbon admixture with the smallest arc time constantin contrary with plasma forming gas Argon where this constant is relatively high (Figure 4). As is very well known, the process of shock waves creation, by pulse current with gradient around $10^{7}-10^{8} \mathrm{~A} / \mathrm{s}$, corresponds to the smallest time constant and creates a highly effective shock wave $[11,13]$ also to deepest disintegration.

TENSIL BOND STRENGTH AND POROSITY DATA MADE BY PRAXAIR INC.

Tensile bond strength between ceramic coatings and stainless steel substrate are within 10,000 - 12, 000 psi without any sub- layer (see Praxair's data below).

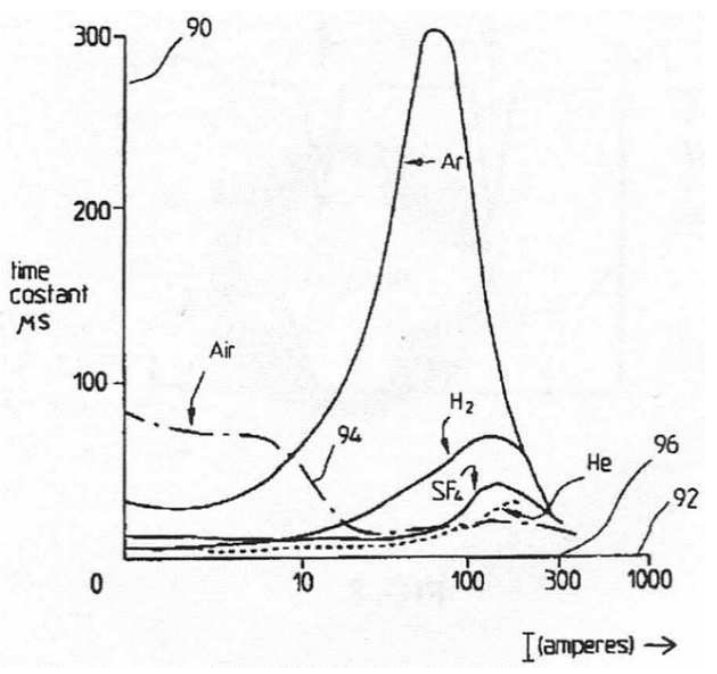

Figure 4. Time us constant of plasma forming gases vs. plasma operation current.

\section{PRAXAIR DATA}

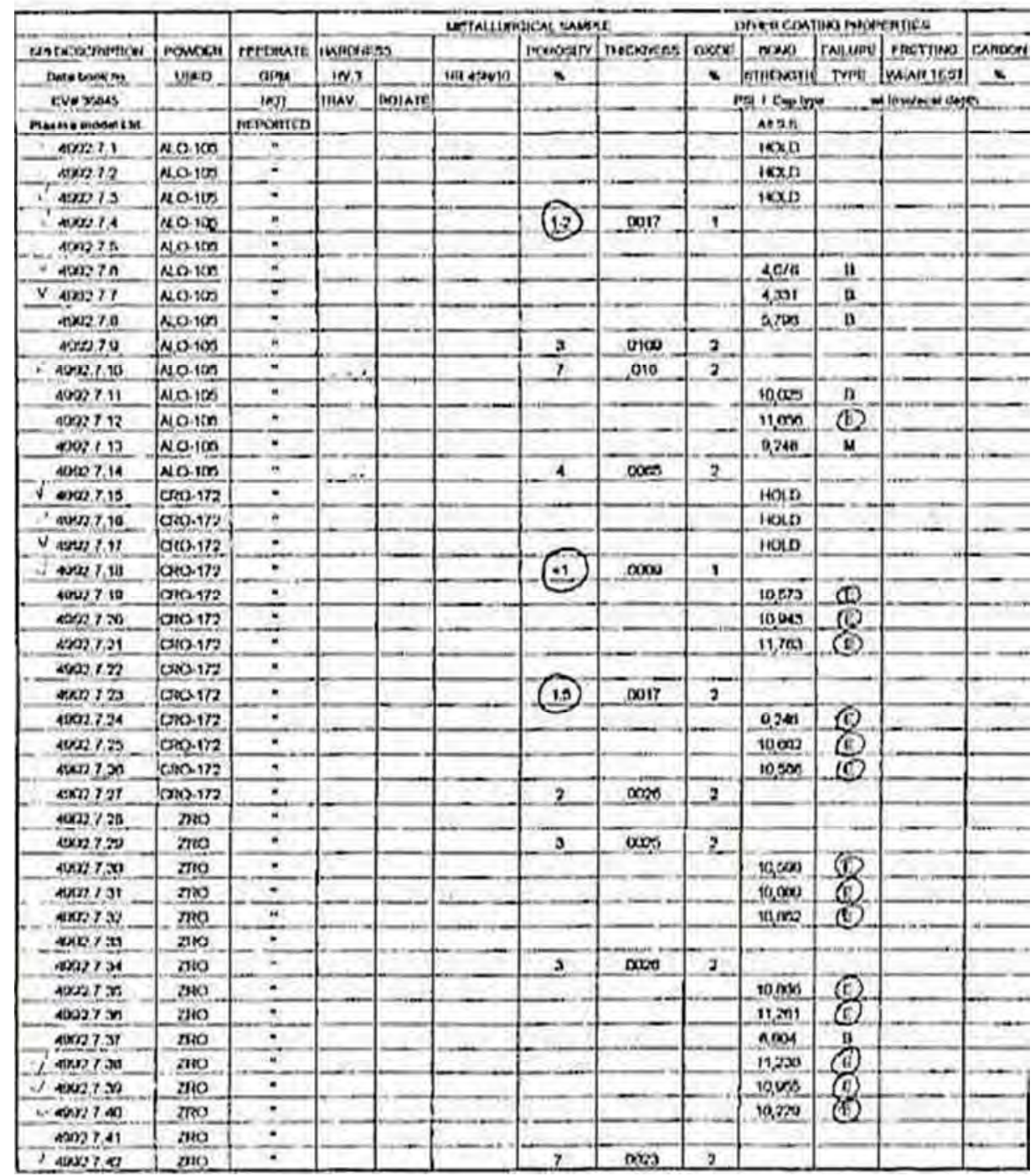

Figure 5. PRAXAIR's data of tensil Bond strenth. 


\section{Conclusion}

Thus, pulse plasma-spraying technology (Figure 6) is demonstrating compelling arguments about significant advantages in comparison with any traditional plasmaspraying one, including production of Nano particles in the modulated Plasma jet:

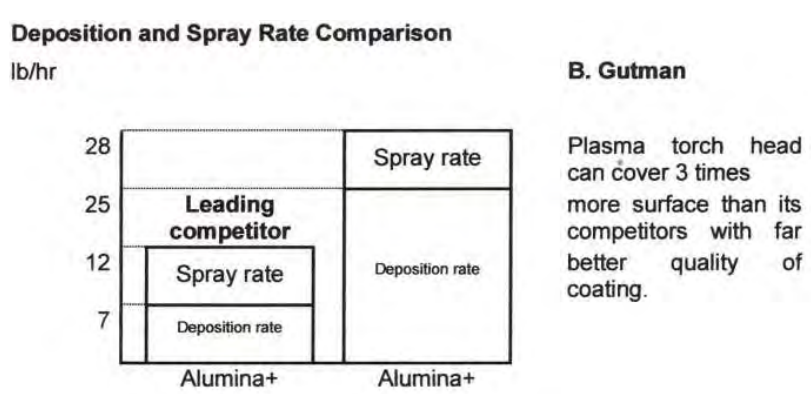

Size of particles sprayed in water

(powder Amdry 6060)

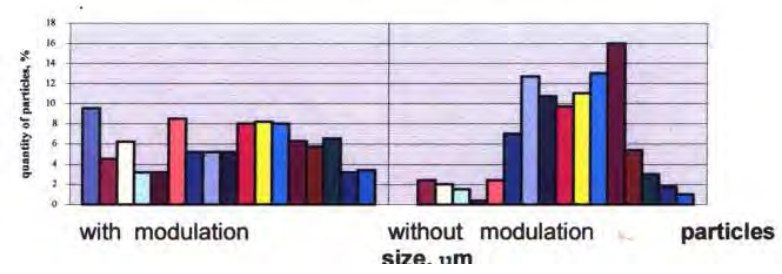

Figure 6. Sizes of sprayed particles nm- $\mu m$ (abscess axes) vs. modulation parameters.

This system is a $75 \mathrm{~kW}$ power DC plasma spray system that provides superior quality of ceramics coatings on surfaces of any size. That is achieved by using pulse's core technology', is the result of almost 30 years of extensive R\&D.

The core technology is based on a high enthalpy plasmachemical reaction involving a modulated plasma Arc within a specially designed torch head. Highest parameters are achieved through a proprietary Arc current modulation scheme and the specially designed torch head. This technology allows controlling the size of spray particles on their way to a target surface.

\section{Deliverables}

1) Unique system allowing to control spray particles' size before they reach target surface

2) Ceramic and other coating cost is 3-10 times less than for leading competitors' products

3) Excellent coating results

4) Highest bond strength of over 80 mega $\mathrm{Pa}$ (without sub layer)

5) Highest deposition rate

6) Highest deposition efficiency

- Patent USA \#5900272, Israel Patent \# 103 069, PCT/US98/22011 International Patent, $[14,15]$.
7) Lowest coating porosity

8) Comparatively low torch noise level

9) Control of the coating porosity, micro hardness, bond strength and density by arc modulation during spraying process

\section{References}

[1] "Method of Supplying of the Plasma Tron." Boris Gutman, Certificate (USSR), \#702934, 13.02, 1978. (First Patent for DC torch modulation)

[2] "Method of Supplying of the DC Arc Plasma Tron." Boris Gutman, Certificate (USSR), \#1227096, 1984.

[3] "Method of Plasma Spraying of Coatings." Boris Gutman, Certificate (USSR), \#1400463, 26.5.86, 1986.

[4] "Method of the Plasma Spraying in DC Arc Plasma Tron." Boris Gutman, Certificate (USSR), \#1400466, 1986.

[5] "Installation for Plasma Detonation Spraying of the Coatings." Boris Gutman, Certificate (USSR) \#1551225, 4.08.1986.

[6] "Method of the Plasma Treatment." Boris Gutman, Certificate (USSR), \#1632670, 15.03.1989.

[7] "Method of Obtaining of the Pyro gas." Boris Gutman, Certificate (USSR), \#1601107, 26.01.1987.

[8] "Method and Devices for Plasma Spraying and/or Plasma Cutting." Boris Gutman, Patent (Israel), \#103069, 26.5.1995.

[9] "Plasma Spraying Arc Current Modulation Method." Priority date: October 27, 1997, US Patent Number: 5,900,272. Date of Patent: May 4, 1999.

[10] "Improved Plasma Spraying Method \& Apparatus, PCT/US 98/22011. Priority date: October 27, 1997, Date of Patent: May 4, 1999.

[11] "Nano plasma technology production for tiles against piercing Weaponry," B. Gutman, "The Open Materials Science Journal", \#, page. 40-46, 2009.

[12] "Shock wave atomization physical mechanisms of modulated DC plasma torch during spraying of coatings", B. Gutman, "Atomization and Spraying”, Journal, Vol. 16, Issue3 (2006).

[13] Pulse modulation spraying technology to produce ceramics materials for fusion and fission reactor, Poster of conference: The Nuclear Materials Conference, 07 - 10 November 2016, Montpellier, France. 